\title{
Evaluación de la rentabilidad económica y captura de carbono en plantaciones de cacao en el plan Chontalpa, Tabasco
}

\section{Evaluation of economic profitability and carbon sequestration in cocoa plantations in the Chontalpa plan, Tabasco.}

\author{
Trinidad Félix, Wilio; Sol Sánchez, Ángel; Galindo Alcántara, \\ Adalberto; Editor Académico Dr. Carlos A. Zuniga González
}

Wilio Trinidad Félix

aga2003a@hotmail.com

División Académica de Ciencias Biológicas, Mexico

Ángel Sol Sánchez

aga2003a@hotmail.com

Colegio de Postgraduados, Campus Tabasco, Mexico

Adalberto Galindo Alcántara

aga2003a@hotmail.com

División Académica de Ciencias Biológicas, Mexico

Editor Académico Dr. Carlos A. Zuniga González

Universidad Nacional Autónoma de Nicaragua, León,

Nicaragua

Revista Iberoamericana de Bioeconomía y Cambio Climático

Universidad Nacional Autónoma de Nicaragua, León, Nicaragua ISSN-e: 2410-7980

Periodicidad: Semestral

vol. 2, núm. 1, 2016

czuniga@ct.unanleon.edu.ni

Recepción: 12 Junio 2015

Aprobación: 18 Diciembre 2015

URL: http://portal.amelica.org/ameli/journal/394/3941750001/

DOI: https://doi.org/10.5377/ribcc.v2i1.5680

Autor de correspondencia: aga2003a@hotmail.com
Resumen: En este estudio se evalúo la rentabilidad económica y la captura de Carbono del sistema agroforestal cacao entre tres parcelas de diferentes edades. Se aplico un cuestionario estructurado a 36 productores pertenecientes al poblado C-34 de Huimanguillo, Tabasco. Se evaluaron los egresos e ingresos en términos monetarios de la plantación del cacao. Para la determinación de la captura de Carbono se estudiaron nueve sitios con superficie de tres hectáreas cada uno pertenecientes al poblado C-34 y un sitio ubicado en el Km-21 del campo experimental del Colegio de Postgraduados. Para la cuantificación de Carbono, se utilizó la metodología rápida para la estimación y monitoreo de captura de carbono propuesta por Rendón y Soto, 2007. Para el análisis estadístico se utilizó un análisis ANOVA y una prueba Tukey Kramer. La plantación de cacao de 20 años obtuvo la mayor utilidad con \$7 653 \pm \$ 3921 , mientras que la plantación de cacao de 30 años obtuvo la menor utilidad promedio con $\$ 5899 \pm \$ 3420$. El análisis estadístico demostró que no hubo diferencias estadísticas significativas en relación al Carbono capturado en las parcelas evaluadas; Sin embargo, en las plantaciones de cacao de 15 años de edad se obtuvo un valor promedio $100.6 \pm 110.5 \mathrm{t} / \mathrm{C} / \mathrm{ha}-1$ mientras que en las de 20 años se obtuvo $49.9 \pm 14$ t/C/ha- 1 .

Palabras clave: Carbono, Cacao, Ingresos, Sistema Agroforestal.

Abstract: The profitability and capture carbon in three cocoa plots from different ages was evaluated. A structured questionnaire was applied a 36 cocoa farmers belonging to the populated C-34 of Huimanguillo, Tabasco. Expenditures and revenues was evaluated in monetary terms of the cacao plantation. For the determination of carbon capture nine sites were studied with surface of three acres each belonging to the populated C-34 and a site located at Km-21 experimental field of the Graduate College. For quantification of carbon, rapid methodology for estimating and monitoring carbon sequestration given by Rendon \& Soto was used, 2007. For statistical analysis, ANOVA analysis was used and Tukey Kramer test. The cocoa plantation of 20 years obtained the most useful with $\$ 7,653 \pm \$ 3,921$, while cocoa plantation 30 years of age had the lowest average income to $\$ 5.899 \pm 3.420$. Statistical analysis showed no statistically significant differences in terms of carbon captured from one age to another. However, Cacao plantations 15 old had the highest average in $\mathrm{t} / \mathrm{ha}-1 \mathrm{de} \mathrm{C}$ (100.6 
\pm 110.5 t/ha-1), while plantations 20 years old had the lowest average catch of $\mathrm{C}(49.9 \pm 14 \mathrm{t} / \mathrm{ha}-1)$.

Keywords: Carbon, Cocoa, Income, Utility, Agroforestry System.

\section{INTRODUCCIÓN}

En el ámbito internacional, los problemas ambientales producto del desarrollo económico, además de la degradación de los recursos naturales generan repercusiones económicas que pueden se mitigadas con el uso de sistemas de producción alternativos como la agroforestería. Esta además de colaborar con la reducción de emisiones de CO2, genera otros beneficios ambientales (la conservación del suelo, conservación de la calidad del agua y conservación de la biodiversidad) y económicos (producción de madera y leña) (Carrascosa 1995; Reitsma et al. 2001; Ortiz 2002; Nair et al. 2009). De igual forma, en México la degradación ambiental ha disminuido el potencial de los servicios ambientales, propiciando además problemas de bajos rendimientos en la producción agropecuaria (CONABIO 2006; SERMARNAT 2006).

La captura de carbono, es uno de los servicios ambientales de relevancia a nivel internacional por su contribución directa a la mitigación del fenómeno cambio climático y el calentamiento global. Por lo que se han desarrollado programas forestales que promueven la captura de CO2 (CONANP 2001). Los sistemas agroforestales como el cacao (Theobroma cacao L.), también pueden ser una alternativa en la reducción de CO2 (Lapeyre et al. 2004). Tabasco es uno de los estados con mayor superficie de cacao a nivel nacional, por lo que resulta interesante evaluar la factibilidad de ser considerado para los programas de captura de CO2 (González 2005).

Los bienes y servicios ecológicos más conocidos son la recarga de los mantos acuíferos y la captura de carbono, de los cuales este último, ha sido sujeto de más estudio por su relación directa con en el cambio climático global (Beer et al. 2003; Silva 2001). Algunas de las alternativas propuestas incluyen el recurrir a actividades forestales y agroforestales para capturar y almacenar el carbono, gas muy importante dentro de la dinámica del calentamiento global (Orrala 2007). Autores como Dixon et al. (1991) y Brown (1998), señalan la importancia de realizar estudios que generen datos de la cantidad de carbono fijada o almacenada en los mismos; cuantificar económicamente su valor y otorgar pago al productor por el servicio ambiental brindado por medio del proyectos llamado mecanismo de desarrollo limpio (Brown 1998).

Por lo tanto el sistema agroforestal de cacao, constituyen una alternativa importante para detener o mitigar la erosión de los recursos naturales. El cultivo del cacao (Theobroma cacao L.) históricamente ha sido una de las especies sometida a explotación en Tabasco por muchos años, aun cuando el mismo no constituye una fuente importante en la dieta alimenticia de la población en general (García 1994), sin embargo constituye una fuente importante de generación de ingresos directos para un grueso número de productores del cultivo, beneficiando económicamente en forma indirecta a otro sector de la población, que procesa y comercializa el producto principal y sus derivados (FAO 2003).

Para el estado de Tabasco, este cultivo representa un renglón de importancia económica y ambiental dentro el proceso de diversificación de cultivos, como tendencia actual y futura en el país y de participación en el mercado externo, donde constantemente se observa la necesidad de satisfacer una demanda que en el ámbito mundial nunca ha sido satisfecha. (Cairns M.A,; et all, 1997)

\section{NotAS DE AUTOR}


Con base en la creciente demanda mundial de participación en el mercado externo, el interés y necesidad de diversificar la producción en el país, más el interés de los productores por el establecimiento de plantaciones comerciales de cacao como sistema agroforestal y la existencia en Tabasco de áreas aptas para el cultivo, es necesario asesorar a agricultores en la planificación de los sistemas productivos orientando el manejo, seguimiento y evaluación de las actividades productivas en el ámbito de la agricultura sostenible y los recursos renovables. (Yuliasmara F; et all, 2009).

Sin embargo, antes de proponer la aplicación de ciertas prácticas agroforestales en un área determinada, es necesario conocer a fondo su situación. Por lo tanto en el presente trabajo se evaluó la rentabilidad económica de algunas plantaciones de Cacao (Theobroma cacao L.) y se determino el potencial de estos sistemas en la captura de Carbono. (Ortiz Pérez M. A; Siebe C; Cram S, 2005)

\section{Materiales Y MÉTODOS}

\section{Área de Estudio}

El presente estudio se realizó en el poblado C-34 (Benito Juárez García) del municipio de Huimanguillo, Tabasco ubicado en las coordenadas 18o 2' N y 93o 24’ W y un sitio ubicado en el Campo Experimental Km-21 del Colegio de Postgraduados en las coordenadas 17o 59’ N y 93o 37’ W (Figura 1).

La zona presenta una influenza directa de las corrientes de aire marítimas proveniente del Golfo de México y que favorece un clima cálido húmedo tropical lluvioso que, según la clasificación de Kôeppen modificado por García (1988), es el del tipo $\mathrm{Am}(\mathrm{f}) \mathrm{w}(\mathrm{i})$, en donde las temperaturas medias, máximas y mínimas oscilan entre 30.3 en el mes de mayo y $20.0^{\circ} \mathrm{C}$ en el mes de enero, las máximas y mínimas absolutas alcanzan hasta $40{ }^{\circ} \mathrm{C}$ y $10^{\circ} \mathrm{C}$ en los mismo meses. En el municipio ocurre una precipitación media anual de $2643 \mathrm{~mm}$ con un promedio máximo mensual de $335 \mathrm{~mm}$, lo que aunado a su orografía lo hace altamente propenso a inundaciones en los meses de mayor precipitación (Hairaah K, et all, 2005).

Fisiográficamente se caracteriza por ser zona plana, formada de depósitos aluviales de edad Cuaternario Reciente (INEGI 2001). Destacan los relieves denominados diques naturales o llanuras aluviales altas de forma convexa (Ortiz et al. 2005) y se caracterizan por presentar sedimentos limosos y arenosos que originan Fluvisoles éutricos, gléyicos y calcáricos, considerados como los suelos más fértiles de Tabasco en donde se establece las plantaciones de Cacao. (IPCC, 2003)

\section{Metodología}

\section{Etapa I. Revisión bibliográfica}

Se realizó una revisión de literatura con carácter científico cuyos estudios están enfocados en la evaluación económica y la captura de carbono en las plantaciones de cacao como Sistema Agroforestal (SAF) y en otros tipos de sistemas productivos.

Etapa II. Selección del Sistema Agroforestal Cacao

Las plantaciones de cacao (Theobroma cacao L.) fueron seleccionados en base aun reconocimiento en campo acompañado del productor. La elección de los cacaotales para el caso de la determinación de la captura de C, se basó en tres edades distintas los cuales son: cacaotal joven o reciente (15 años), cacaotal en producción plena (20 años) y cacaotal en abandono productivo (30 años) (López 1987; Somarriba et al. 2005).

\section{Etapa III. Evaluación de los reservorios de carbono}

Para la cuantificación de carbono $\mathrm{C}$ se seleccionaron tres productores por cada edad generándose un total de nueve productores, la extensión de terreno de cada productor fue de 3 ha, generándose un total de 27 ha en la cuales se realizo el siguiente estudio.

Para la medición de la biomasa aérea se empleó la metodología rápida para la estimación y monitoreo de captura de carbono (Rendón \& Soto 2007) la cual esta diseñada para emplearse en una superficie total de 1 ha. La medición de los diferentes componentes fue realizada de la siguiente manera. 
Arboles adultos: En el centro de la plantación de cada cacaotal se delimitaron dos rectángulos que forman entre ellos una "T" cada rectángulo media $20 \mathrm{~m}$ de largo por $12.5 \mathrm{~m}$ de ancho (en total $500 \mathrm{~m} 2$ ). En cada uno de los rectángulos se registró y se midió el diámetro de todos los árboles mayores a $10 \mathrm{~cm}$. Esta medida se tomó a la altura de pecho con la ayuda de una cinta diamétrica marca Forestry Suppliers. También se midió la altura con la ayuda de una vara $(3 \mathrm{~m})$ y un clinómetro marca Brunton con la fórmula; Altura $(\mathrm{H})=($ Angulo de arriba + Angulo de la base) x Distancia Horizontal/100.

Medición de los juveniles: En la mitad de uno de los rectángulos (un cuarto de la superficie total equivalente a $125 \mathrm{~m} 2$ ) se midieron con la ayuda de un vernier todos los arboles juveniles que midieron más de $2.5 \mathrm{~cm}$ pero menos de $9.9 \mathrm{~cm}$ de diámetro, también se registro la altura. Herbáceas: Para la medición de este componente únicamente fue necesario medir la altura de las plantas y apreciar a simple vista el \% que cubre del suelo (Redon \& Soto 2007), por lo cual fue necesario estimar en doce unidades de $0.25 \mathrm{~m} 2$ la cobertura visual y registrar si la mayoría de tallos son leñosos o carnosos. Las doce unidades se distribuyeron al azar, en cada unidad se midió y se anoto la altura máxima de las hierbas y la altura predominante (Hdom) de las hierbas o plántulas presentes (altura modal) y se obtuvo un promedio con ambas alturas.

Hojarasca y humus: Dentro de las unidades de $0.25 \mathrm{~m} 2$ se midió la profundidad de la hojarasca y humus en un punto al azar introduciendo un flexómetro. De este modo se calculo un promedio para estimar el peso seco de la hojarasca y humus (Redon \& Soto 2007). Los información obtenida de cada parcela del muestreo fue recolectado en hojas de campo.

Para determinar la densidad de la madera se empleó la lista de las densidades de las especies de árboles tropicales propuesto por Brown (1997), la base de datos de Zanne et al. 2009. y algunos datos de páginas electrónicas. Con los datos obtenidos anteriormente se calculó la biomasa aérea de los arboles adultos y la biomasa de los diferentes partes de la planta utilizando la siguientes formulas (Cuadro 1 y 2 ).

CUADRON ${ }^{\circ} 1$

Ecuaciones para estimar la Biomasa ( $\mathrm{kg} /$ árbol)

\begin{tabular}{lll}
\hline Especies de arboles & Ecuaciones Alometricas & Autor \\
\hline Arboles forestal & $\mathrm{Y}=\exp \left(-2.9777+\ln \left(\mathrm{pD}^{2} \mathrm{~h}\right)\right)$ & Chave et al. 2005 \\
Cacao & $\mathrm{Y}=0.1208 \mathrm{D}^{1.98}$ & Yuliasmara, 2008 \\
Naranja & $\mathrm{Y}=0.27 \mathrm{AB}+0.000514(\mathrm{AB})^{2}-6.64$ & $\mathrm{IPCC}, 2003$ \\
Plátano & $\mathrm{Y}=0.303 \mathrm{D}^{2.1345}$ & Hairiah et al. 2005 \\
\hline
\end{tabular}

$\mathrm{Y}=$ biomasa $(\mathrm{kg} /$ árbol), $\mathrm{p}=$ densidad por especie o promedio $(\mathrm{gr} / \mathrm{cm} 3)$,

$\mathrm{D}=$ diámetro a la altura del pecho $(\mathrm{cm}), \mathrm{h}=$ altura, $\mathrm{AB}=$ área basal $(\mathrm{cm} 2)$.

El carbono total será la mitad de la suma de todos los componentes, ya que convencionalmente se considera el factor de proporcionalidad del $50 \%$ para obtener el carbono total contenido en la biomasa viva (IPPC 2001).

El resultado debe expresarse en toneladas de carbono $(\mathrm{tC})$, haciendo las conversiones correspondientes y dicho resultado extrapolarse a hectáreas para que el resultado sea dado en $\mathrm{t} / \mathrm{C} / \mathrm{ha}$. 


\begin{tabular}{lll} 
& \multicolumn{2}{c}{$\begin{array}{c}\text { CUADRO N² } \\
\text { Ecuaciones para la determinación de la biomasa de los } \\
\text { diferentes compartimientos dentro de un SAF-Cacao. }\end{array}$} \\
\hline Biomasa & Ecuaciones & Autor \\
\hline Raíz & $\mathrm{BR}=\exp \left(-1.0587+0.8836^{*} \mathrm{LN}(\mathrm{ABD})\right)$ & Cairns et al. 1997 \\
Herbáceas & $\mathrm{B}_{\text {herbaceass }}=3.33 \mathrm{x}_{5}$ & Chave et al. 2005 \\
Hojarasca y humus & $\mathrm{B}_{\mathrm{H}}=40 \mathrm{H}_{\mathrm{p}}$ & PCC, 2003 \\
\hline
\end{tabular}

\footnotetext{
$\mathrm{BR}=$ Biomasa total de raíces gruesas y finas $(\mathrm{t} / \mathrm{ha}) . \operatorname{Exp}(\mathrm{n})=2.718 \mathrm{n} . \mathrm{ABD}=$ Biomasa arbórea más arbustiva $(\mathrm{Mg} / \mathrm{ha}$ de materia seca). Bherbáceas $=$ Biomasa de herbáceas promedio por hectárea $(\mathrm{kg} / \mathrm{ha})$. 3.33 = factor de conversión, $\mathrm{xs}=$ peso seco promedio por cuadro $(\mathrm{gr} / \mathrm{m} 2) . \mathrm{BH}=$ Biomasa de hojarasca $(\mathrm{kg} /$ ha). $40=$ Factor de conversión (para convertir a kg/ha). HP =Biomasa promedio por cuadro $(0.25 \mathrm{~m} 2)$.
}

\section{Etapa IV. Diversidad de especie}

Se emplearon los siguientes índices para conocer la diversidad un cacaotal con respecto a otro (Cuadro 3).

$$
\begin{array}{ll}
\multicolumn{2}{c}{\begin{array}{c}
\text { CUADRO } \mathrm{N}^{\circ} 3 \\
\text { Índice de diversidad y Equitatibilidad. }
\end{array}} \\
\begin{array}{ll}
\hline \text { Índices } & \text { Fórmula } \\
\hline \text { Shannon-Wiener } & \mathrm{H}^{\prime}=-\sum P i * \ln P i \\
\text { índice de equidad } & \mathrm{E}=\mathrm{H}^{\prime} / \ln \mathrm{S} \\
\hline
\end{array} \\
\end{array}
$$

\begin{tabular}{ll}
\hline Índices & Fórmula \\
\hline Shannon-Wiener & $\mathrm{H}^{\prime}=-\sum P i * \ln P i$ \\
índice de equidad & $\mathrm{E}=\mathrm{H}^{\prime} / \ln \mathrm{S}$ \\
\hline
\end{tabular}

\section{Etapa V. Aplicación de encuesta a productores de Cacao}

Para realizar el análisis económico fue necesario conocer en número de productores de cacao registrados en el poblado C-34 (Benito Juárez García). Sin embargo solo se tomaron 80 productores con plantaciones cuya extensión de terreno era mayor o igual a una ha y cuyas plantaciones estuvieran inmersa en las tipologías de cacaotal joven, en producción y en abandono productivo. La determinación de la muestra se llevo acabo con el método de muestreo aleatorio simple para un tamaño de población finita usando la siguiente formula estadística:

$$
n=\mathrm{Z}^{2} \mathrm{pqN} / \mathrm{N} e^{2}+p q
$$

\section{Donde:}

$\mathrm{n}=$ tamaño de la muestra. $\mathrm{N}$ = tamaño de la población. $\mathrm{p}=$ probabilidad a favor. $\mathrm{q}=$ probabilidad en contra. $\mathrm{Z}=$ nivel de confianza. $\mathrm{e}=$ error de estimación.

Asignándosele a p y q la probabilidad de 0.5 respectivamente con un nivel de confianza del $90 \%$ (equivale a 1.645) y un error de estimación del 10\%. [H1]A partir de ello se obtuvo un total de 36 productores a encuestar, a los cuales se les aplico el cuestionario estructurado durante los meses de Mayo y Junio del 2012.

\section{Etapa VI. Análisis de los datos}

Los datos obtenidos del muestreo para la estimación de la captura de carbono fueron capturados en una base de datos de Excel y se realizaron los cálculos en base a las fórmulas antes descritas. Mediante operaciones matemáticas simples, se extrapoló la captura de carbono a t/ha. Posteriormente se calculó promedio y desviación estándar de variables sobresalientes con respecto a componentes esenciales en la determinación de la captura de C. 
Los datos obtenidos de las encuesta fueron capturados en una base de datos de Excel 2010. En el análisis de rentabilidad se estudiaron los gastos y los ingresos en término monetario del SAF- Cacao. Se determinaron los datos necesarios de los insumos, productos y precios. Se utilizaron los precios de la mano de obra contratada y los insumos comprados. Los ingresos se obtuvieron de los productos para la venta. A partir de estos datos se obtuvo la utilidad (mediante operaciones matemáticas simple) que los cacaotales brindan a los productores.

Para determinar diferencias estadística entre la captura de $C$ con respecto a la edad y de la utilidad con respecto a la edad se utilizó un ANOVA, y un test a posteriori de Tukey Kramer HSD

\section{RESULTADOS}

\section{Diversidad de arboles en las plantaciones de Cacao}

Cacaotal de 15 años: se registraron encontró 100 individuos pertenecientes a 13 especies. Las más abundantes fueron Erythrina americana Mill, Gliricidia sepium (Jacq.) Steudel y Diphysa robinioides Benth. En menor proporción se encontró Artocarpus altilis (Parkison) Fosberg y Guazuma ulmifolia Lam (Cuadro 4).

\section{CUADRO $\mathrm{N}^{\circ} 4$}

Especies asociadas a las plantaciones de Cacao (Theobroma cacao).

\begin{tabular}{|c|c|c|c|c|c|}
\hline & Nombre Cientifico & *Usos & 15 & 20 & 30 \\
\hline Guanábana & Annona muricata $\mathrm{L}$. & F & 0 & 0 & 1 \\
\hline Castaña & Artocarpus altilis (Parkison) Fosberg. & F & 1 & 2 & 0 \\
\hline Hule & Castilla slastica Sessé. & M & 0 & 0 & 2 \\
\hline Cedro & Cedrela odorata $\mathrm{L}$. & M & 6 & 6 & 2 \\
\hline Naranja & Citrus sinensis $\mathrm{L}$. & F & 2 & 2 & 0 \\
\hline Tatúan & Colubrina arborescens (Mill.) Sarg. & $\mathrm{M}$ & 6 & 12 & 11 \\
\hline Caracolillo & Ormosia macrocalyx Ducke. & M & 0 & 0 & 1 \\
\hline Chilpicohite & Diphysa robinioides Benth. & s & 9 & 10 & 12 \\
\hline Mote & Erythrina americana Miller. & s & 42 & 100 & 31 \\
\hline Pito & Erythrina berteroana Urban. & s & 3 & 8 & 1 \\
\hline Cocohite & Gliricidia sepium (Jacq.) Steudel. & S & 11 & 0 & 0 \\
\hline Melina & Gmelina arborea Roxb. & M & 8 & 0 & 0 \\
\hline Guácimo & Guaruma ulmifolia Lam. & M & 1 & 3 & 2 \\
\hline Majahua & Hampea trilobata Standley. & S & 0 & 0 & 11 \\
\hline Cedrillo & Trichilia hirta L. & M & 4 & 0 & 0 \\
\hline Mango & Mangifera indica $\mathrm{L}$ & F & 0 & 0 & 1 \\
\hline Plátano & Musa paradisiaca $\mathrm{L}$. & F & 3 & 0 & 1 \\
\hline Aguacate & Persea americana Miller. & F & 4 & 2 & 0 \\
\hline Mamey & Mammea americana $\mathrm{L}$. & F & 0 & 0 & 1 \\
\hline Caoba & Swietenia macrophylla King & M & 0 & 0 & 1 \\
\hline \multirow[t]{4}{*}{ Macuili } & Tabebuia rosea (Bertol.) DC. & M & 0 & 4 & 1 \\
\hline & Total & & 100 & 149 & 68 \\
\hline & $\mathrm{H}^{\prime}$ & & 2.002 & 1.288 & 1.924 \\
\hline & $\mathrm{E}$ & & 0.759 & 0.559 & 0.729 \\
\hline
\end{tabular}

$\mathrm{H}^{\prime}=$ índice de diversidad de Shannon-Wiener. E= Índice de equitatibilidad. * Usos: Frutales (F), Maderable (M), y Sombra (S). 
Cacaotal de 20 años: se encontró un total de 149 individuos agrupados en 10 especies, entre la más abundante destaca Erythrina americana Miller, seguida de Colubrina arborescens (Mill.) Sarg. y Diphysa robinioides Benth. En menor proporción encontramos Persea americana Miller, Citrus sinensis L. y Artocarpus altilis (Parkison) Fosberg.

Cacaotal de 30 años: se encontró un total de 79 individuos pertenecientes a 15 especies, entre la más abundante tenemos a Erythrina americana Miller, seguida de Diphysa robinioides Benth y Colubrina arborescens (Mill.) Sarg. En menor proporción se encontró a Annona muricata L., Erythrina berteroana Urban, Mangifera indica L., Musa paradisiaca L., Mammea americana L., Sideroxylon gaumeri Pitt, Swietenia macrophylla King y Tabebuia rosea (Bertol.) DC.

De acuerdo con el índice de Shannon el cacaotal de 15 años presentó el índice de diversidad más alto con un valor de 2.0, posterormente el cacaotal de 30 años de edad con 1.9, mientras que el menor lo presenta el cacaotal de 20 años de edad con 1.28. Según estos resultados el cacaotal de 15 años presenta una estructura con mayor equidad y diversidad.

A pesar de la similitud en los valores del cacaotal de 20 y 30 años de edad, existe una alta diferencia en diversidad en las zonas estudiadas, pues los valores del índice de diversidad de Shannon varían generalmente de 1,5 a 3,5 (Zak y Willig, 2004).

Captura de carbono en los diversos compartimientos del SAF-Cacao

A partir de la metodología empleada en las plantaciones de cacao $(15,20$ y 30 años) para la determinación de captura de $\mathrm{C}$, se encontró que la biomasa en raíz del cacaotal de 15 años presenta el mayor promedio con $26.9 \mathrm{t} / \mathrm{ha}$. El menor promedio se presenta en el cacaotal de 30 años con $12.9 \mathrm{t} / \mathrm{ha}$ (Cuadro 5).

Las plantaciones de 20 y 30 años presentan el mayor promedio en biomasa de hojarasca y humus, las de 15 años son de menor promedio, este resultado en la plantación de 20 años podría estar originándose probablemente a las prácticas de manejo como las podas el cual contribuye a una mayor producción de biomasa, en cambio en la de 30 años es claro que esta relacionado con la edad y la estructura de sus ramas de cada especie. Las plantaciones de 30 años presenta el mayor promedio en biomasa de herbáceas, mientras que el menor promedio lo presenta el cacaotal de 20 años (Cuadro 5).

\section{CUADRO No5}

Biomasa ( $t / h a)$ presente en tres componentes del SAF-Cacao.

\begin{tabular}{ccccccc}
\hline & \multicolumn{2}{c}{ Raíz } & \multicolumn{2}{c}{ Hojarasca y Humus } & \multicolumn{2}{c}{ Herbáceas } \\
\cline { 2 - 7 } Edad (años) & $\bar{x}$ & $\sigma$ & $\bar{x}$ & $\sigma$ & $\bar{x}$ & $\sigma$ \\
\hline 15 & 27.8 & 31.21 & 20.8 & 4.8 & 0.5 & 0.5 \\
20 & 13.5 & 4.4 & 22.4 & 2.8 & 0.2 & 0.1 \\
30 & 12.9 & 15.9 & 22.4 & 2.8 & 0.5 & 0.3 \\
\hline
\end{tabular}

$\mathrm{x} \#=$ promedio. $\sigma=$ desviación estándar

Los arboles de cacao de 15 años de edad presentan el mayor promedio de biomasa, los cacaotales de 30 años de edad presentan el mayor promedio en altura, mientras que los de 20 años son los de menor promedio en altura y biomasa (Cuadro 6).

Las especies asociadas a los arboles de cacao de 15 años de edad presenta el mayor promedio en altura, diámetro y biomasa, mientras que el de 20 años de edad presentan el menor promedio en altura, diámetro y biomasa. 


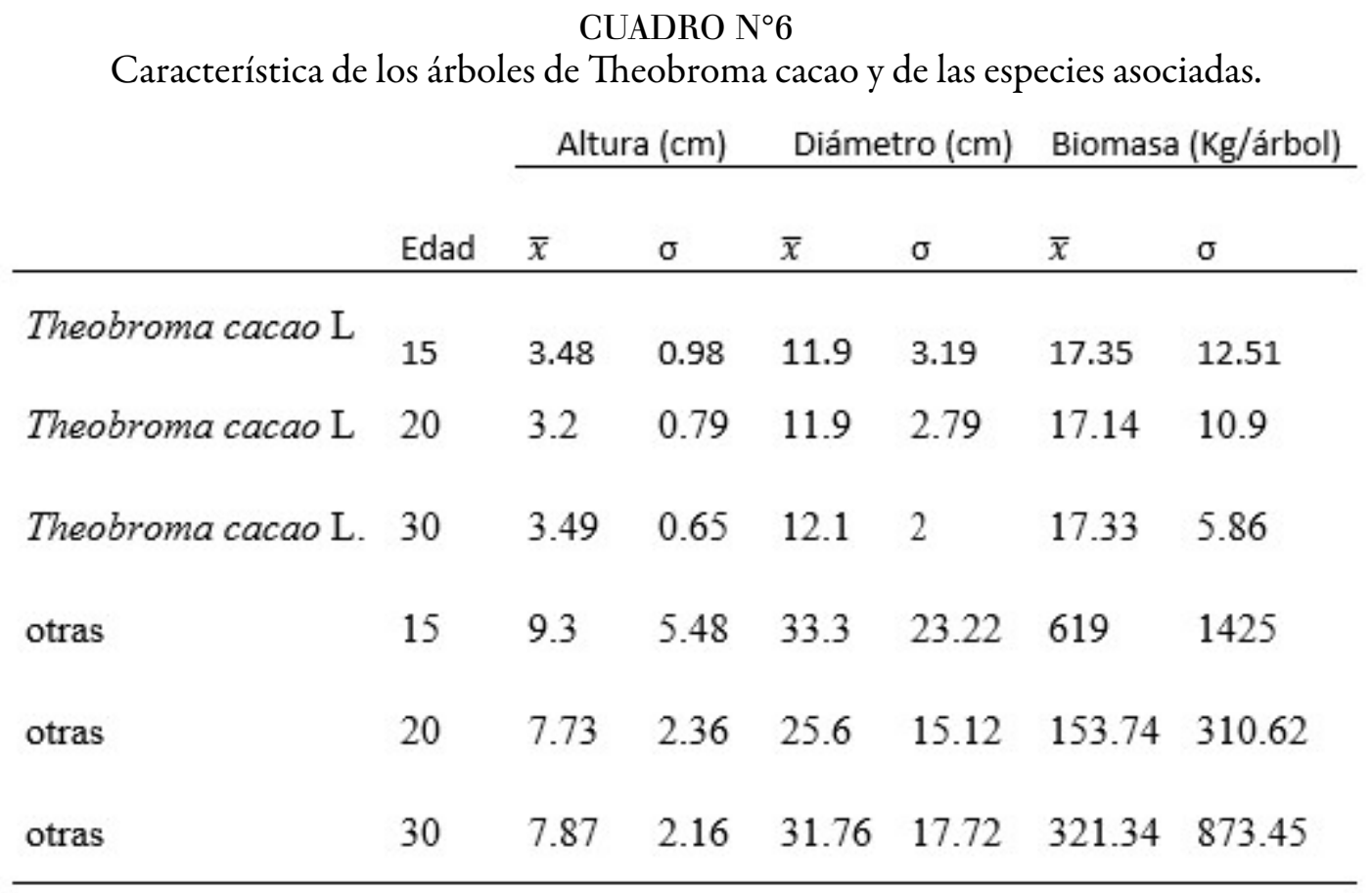

x\#=promedio. $\sigma=$ desviación estándar

Al momento de cuantificar la cantidad de carbono total para las plantaciones de Cacao (Theobroma Cacao L.), se obtuvo que las plantaciones de cacao con edad de 15 años presentan el mayor promedio con 100.6 en t/ha- 1 de C, los de 20 presenta el menor promedio con 49.9 t/ha-1 de C. Sin embargo al generar el análisis estadístico no se encontraron diferencia estadística significativa $(p=0.05 \%$, test de Tukey-Kramer, entre los sitios muestreados.

\section{Caracterización del los productores con respecto al SAF-Cacao}

A partir de los datos analizados de las encuesta se encontró que el $78 \%$ de los productores tienen pequeña propiedad mientras que el resto es ejidal.

El $78 \%$ de los productores asocian arboles de sombra a las plantaciones de cacao. De acuerdo con los productores los principales arboles utilizados son Diphysa rubinioides Benth, Erythrina berteroana Urban, Cedrela odorata L., Gmelina arborea Roxb., Tabebuia rosea (Bertol.) DC., Erythrina americana Miller y Colubrina arborescens (Mill.) Sarg., los cuales recomiendan plantarse un año antes del establecimiento del cacao. La distancia de siembra entre arboles de sombra generalmente de $4 \mathrm{~m} \times 4 \mathrm{~m}$. Sin embargo después de los siete años de establecida la plantación los productores comienzan con la eliminación de algunos árboles de sombra de tal manera que queden a una distancia de $8 \times 8$. Esto con motivo de que la plantación debe de quedar con el $50 \%$ de sombra para que se mantenga en buen estado y evitar la incidencias de plagas y enfermedades. Estos resultado coinciden con los reportados por López et al. 1988; Córdova-Ávalos et al. 2001 en el que menciona que las especies mas utilizada son Diphysa rubinioides Benth, Erythrina berteroana Urban, Cedrela odorata L y Erythrina americana Miller. (García E, 1988)

El número de personas de la familia que participan en las actividades que requiere la producción de cacao en promedio es de tres, mientras que el número de personas contratadas en promedio es de dos.

El $44 \%$ de los productores mencionan que realiza dos cosechas al año los cuales abarca los meses de Octubre - Marzo. Siendo los meses de Octubre, Noviembre y Diciembre la primera cosecha y la que brinda mayores beneficios económicos, puesto que su rendimiento en grano es mayor con respecto a la segunda cosecha la cual corresponde a los meses restantes, estos resultados coincide con lo reportados por Córdova-Ávalos et al. 2001 en el que menciona que la cosecha inicia en el mes de septiembre y termina en el mes de mayo. El $22 \%$ de los 
productores vende su cacao en baba y en seco, mientras que el resto prefiere venderlo en baba solamente. El rendimiento promedio del cacao en baba es de $894 \mathrm{~kg} / \mathrm{ha} \pm 180 \mathrm{~kg} / \mathrm{ha}$, mientras el rendimiento en promedio de cacao seco es de $279 \mathrm{~kg} / \mathrm{ha} \pm 19 \mathrm{~kg} / \mathrm{ha}$. El Precio por kg de cacao en baba generalmente fue de diez pesos. El rendimiento promedio del cacao ya sea en seco o en baba varia en relación a la edad del cacaotal y al tipo de manejo productivo que se le este dando, lo cual concuerdo con Córdova et al. 2001, el cual menciona que la producción por hectárea varía en relación inversa a la edad y al tipo de manejo de las plantaciones.

Para la cosecha y venta del cacao el $89 \%$ de los productores usa como medio de transporte el humano (incluye el uso carretilla, bicicletas y triciclos) y el $11 \%$ hace uso de motocicletas y camionetas para transportar sus productos.

De manera general los productores en su totalidad expresaron que la enfermedad que provoca mayor perdida en la época de lluvias son la Mancha negra (Phytophtora capcisi) que provoca infección y muerte de los frutos. Para evitar dicho daños los cacaoteros realizan aspersiones de oxicloruro de cobre o caldo bordelés (compuesto de sulfato de cobre, cal y agua) en dosis de $1-3 \mathrm{~kg}$ en 200 litros de agua por ha y la otra enfermedad es la Moniliasis (Moniliophtora roreri), las cuales afectan gravemente el rendimiento de su producción, siendo esta ultima difícil de combatirla debido al desconocimiento y falta de capacitación de los productores. Para el control de la mancha negra se lleva acabo en promedio durante tres días por dos veces al año, especialmente en la época de norte cuando la temperatura disminuye y la humedad relativa aumenta. Estos resultados están relacionados con lo reportado por Córdova-Ávalos et al. 2001, en el que menciona que el cultivo de cacao se ve afectado en la época de lluvias son la mancha negra (Phytophtora capcisi) y para controlarlo emplea oxicloruro de cobre. (Callo Concha D. L, et all, 2001)

El $100 \%$ de los productores mencionaron como responsables frecuentes de daño al cultivo a la ardilla negra (Sciurus aerogaster) y el pájaro carpintero (Melanerpes aurifrons), sin embargo para esta plaga comentaron que su control no es posible porque no tienen conocimiento de cómo alejarlo de sus parcelas, lo que coincide con lo reportado por Córdova-Ávalos et al. 2001 en el que menciona como plagas principales y de difícil control a la ardilla negra y el pájaro carpintero.

El control de la lengua de vaca (Syngonium podophyllum) se hace con machete y garabato, este resultado coincide con lo reportado por Cartagena (2010) en el que menciona qua la utilización de materiales para el cultivo no está estandarizado, utilizan la herramienta que tengan a la mano siendo el machete el más común de las herramientas pero sin llegar a generalizarse su uso.

Para el caso de la poda se realiza en promedio durante tres días por una vez al año, consiste en eliminar ramas secas, las enfermas y las entrecruzadas. Los fertilizantes usados son los foliares y se aplican para refrescar a la planta en los meses de sequía, la aplicación de fertilizante se realiza en promedio durante tres días por una vez al año, este resultado coincide por lo reportado por González (2005) en el que menciona que los productores utilizan sus propios recursos para la fertilización, utilizando foliares y no fertilizantes para el suelo lo que limita no solo el rendimiento por hectárea sino también la productividad.

El control de sombra se realiza en promedio durante dos días por una vez al año. De manera general los productores comentan que los arboles como la Erythrina americana Miller, Gliricidia sepium (Jacq.) Steudel y Diphysa robinioides Benth son fáciles de podar y son considerados como sombras refrescantes, contrariamente a la Ormosia macrocalyx Ducke que son especies difíciles de manejar, por el cual prefieren eliminarlos haciéndoles el cinchado. (Chave J, et all, 2005)

Para la cosecha del cacao el promedio de uso de mano de obra es de dos personas, la frecuencia de cosecha varia de tres a cuatro días para evitar el robo de las mazorcas y disminuir el pago de jornal, estos resultados coincide con lo reportado con por Córdova Ávalos et al. 2001, para la cosecha el uso de mano de obra es de 8 a 15 personas, existe el problema del robo, por lo que el productor cosecha los frutos cuando están iniciando su madurez.

Análisis de rentabilidad del SAF-Cacao 
Categorizando a la plantación cacao tomando como variable la edad para determinar la utilidad se encontró lo siguiente:,El mayor costo promedio para el control de maleza, poda, fertilización, cosecha, plagas, y enfermedades se presenta en la plantación de cacao de 20 años de edad, el menor costo promedio para el control de maleza, poda, fertilización y cosecha se presenta en el cacaotal de 15 años de edad (Cuadro 7).

\section{CUADRO N7}

Costo en control de actividades correspondiente a la mano de obra

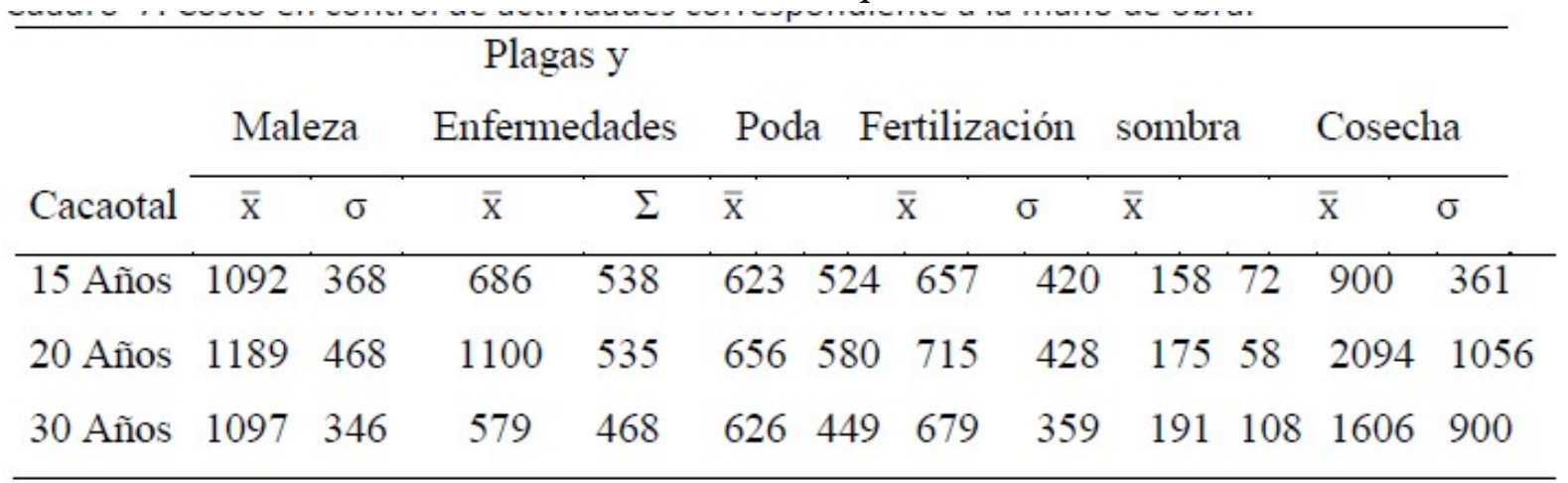

$\mathrm{x} \#=$ promedio. $\sigma=$ desviación estándar.

El cacaotal de 15 años genera una utilidad promedio de \$ $6930 \pm 3386$ pesos, el de 20 años presenta una utilidad promedio de $\$ 7653 \pm 3921$ y el de 30 años $\$ 5899 \pm 3420$ pesos (Cuadro 8). Al generar el análisis estadístico se encontraron diferencias estadísticas significativas ( $\mathrm{p}=0.05 \%$, test de Tukey-Kramer, JMP 2005) entre la utilidad de un nivel de edad a otro en las plantaciones de 15 a 20, de 15 a 30 y de 20 a 30 años de edad. Es importante resaltar que la plantación de 20 años de edad es la que genera la mayor utilidad promedio. La plantación de30 años es la de menores rendimientos. La diferencia de una plantación a otra en cuanto a la utilidad se justifica a la no equidad en cuanto al mantenimiento de la planta de cacao y esto hace que no todos obtengan similar producción por hectárea y por ende iguales ingresos por hectárea derivados de este cultivo. (cuadro n ${ }^{\circ} 8$ )

\section{CUADRO N ${ }^{\circ} 8$}

Utilidad, Ingreso total, Costo total de mano de obra e insumos en las plantaciones de Cacao

\begin{tabular}{|c|c|c|c|c|c|c|c|c|}
\hline \multirow[b]{2}{*}{ Edad } & \multicolumn{2}{|c|}{ Ingreso Total } & \multicolumn{2}{|c|}{$\begin{array}{c}\text { Consumo total de la mano } \\
\text { de obra }\end{array}$} & \multicolumn{2}{|c|}{$\begin{array}{l}\text { Costo total de } \\
\text { Insumos }\end{array}$} & \multicolumn{2}{|c|}{ Utilidad } \\
\hline & $\bar{x}$ & $\sigma$ & $\bar{x}$ & $\sigma$ & $\bar{x}$ & $\sigma$ & $\bar{x}$ & $\sigma$ \\
\hline 15 años & 11856 & 5071 & 4612 & 2045 & 346 & 532 & 6930 & 3386 \\
\hline 20 años & 13230 & 4697 & 5250 & 2266 & 565 & 412 & 7653 & 3921 \\
\hline 30 años & 10819 & 4672 & 4519 & 1790 & 610 & 524 & 5899 & 3420 \\
\hline
\end{tabular}

$\mathrm{x} \#=$ promedio. $\sigma=$ desviación estándar.

El cacaotal abandonado es el que presenta la menor utilidad promedio, dado que la edad de la plantación, el mal manejo productivo, la capacitación técnica del productor y organización influyen en el rendimiento del sistema productivo (Córdova et al 2001). El mayor de insumos lo presenta el cacaotal de 30 años de edad, mientras que el cacaotal de 15 años presenta el menor costo. 


\section{DISCUSIÓN}

Respecto al índice de diversidad y equidad el mayor índice se presenta en la plantación de 15 años, esto se debe a que es una plantación joven y no se han aprovechado los arboles de sombra, hay mayor ingreso de luz del sol y el banco de germoplasma presente puede expresarse en forma más completa. Sin embargo la riqueza y la equidad en la plantación de 30 años, tiende a ser superior con respecto a la de 20 años, dicho resultado podría estar originado puesto que al estar en abandono, permite el crecimiento de otras especies ajenas al árbol de cacao por diversos factores como espacios abiertos por el aprovechamiento maderable de especies como el chipilcó, muerte de árboles frutales como el aguacate y el Chinín lo que permite que los rayos del sol entren al sotobosque y permita que algunas de las semillas el banco de germoplasma germinen y se desarrollen. En ambos casos esta información guarda estrecha relación con los datos obtenidos en la misma región por Sánchez et., al 2012.

De las 21 especies encontradas en las plantaciones de cacao el $76 \%$ son especies frutales y maderables lo que da una clara idea de que el productor diversifica su plantación con especies que generan beneficios económicos y alimenticios. La mayor cantidad promedio de biomasa en t/ha-1 en Hojarasca y Humus está dada por el cacaotal de 20 y 30 años, probablemente se deba que al ser arboles que han alcanzado su máximo crecimiento fisiológico, la ramificación de su estructura y la aportación o caída de las hojas al suelo tanto arboles de cacao, como las especies asociadas, es mayor, este resultado coincide con lo reportado por Lapeyre et al. (2004) en donde menciona que estos resultados pueden deberse a las diferentes edades y la diversidad de especies heterogénea, en donde se desarrollan cada sistema agroforestal.

Las herbáceas presentes en la plantación de 30 años de edad generan una mayor aportación de biomasa en t/ha-1, en comparación con las herbáceas de la plantación de 15 y de 20 años de edad. No obstante la aportación de esta biomasa en la cuantificación del carbono total resulta ser mínima puesto que no tiene leño.

La mayor cantidad de C promedio en ton/ha-1 se ve reflejado en un cacaotal joven (15 años) probablemente a la presencia de arboles forestales como es la Gmelina arborea Roxb., Trichilia hirta L. y Cedrela odorata, estos árboles generalmente están sembrados antes de establecimiento del cultivo de cacao por lo que son arboles de diámetro y altura sobresalientes. En base a la fisiología de la planta de cacao en los sistemas agroforestales de 15 años, la especie de cacao ha alcanzado el máximo potencial de almacenamiento de carbono, llegándose a incrementar aún más estos flujos de $\mathrm{C}$ con el crecimiento de las especies de sombra. Estos flujos generan servicios ambientales y valores aditivos por la producción de madera, frutos, leña, entre otros. La menor captura promedio de C lo presenta el cacaotal en producción plena, probablemente se deba que al ser un sistema que esta manejado por el agricultor, gran parte de las especies de sombra y maderable con gran tamaño en diámetro y altura han sido eliminadas, estos resultados están relacionados con lo citado por Alegre et al. 2001, que los flujos expresan el dinamismo de la acumulación de carbono que varia con el tiempo de vida y el tipo de asociación de cada sistema agroforestal; mientras que el crecimiento de cada especies, varían dependiendo si son forestales y/o frutales.

El 78\% de los productores cuenta con pequeña propiedad, lo que se traduce que existe la posibilidad de que se realicen actividades de mejora en el mismo.

Más del 50\% de los productores encuestados asocian su plantación de cacao con árboles de sombra, lo que permite que los arboles de cacao se desarrollen plenamente al mismo tiempo que le proporciona madera y leña para autoabastecerse.

El número de personas contratadas para el cultivo de cacao se ve reducida por el poco ingreso económico con que cuenta el productor.

El $22 \%$ vende su cacao en seco, mientras que el $78 \%$ de los productores prefiere vender su cacao en baba por la facilidad y rapidez que encuentra en comercializarlo, con el cual se evitan el tiempo de cuidado al secarlo y reducen el gasto económico que implicaría en el secado del mismo. Sin embargo el pago que reciben es bajo 
en comparación si optaran por secarlo, el precio de este varía de 30 a 40 pesos por $\mathrm{kg}$, dependiendo a donde lo comercializan.

\section{CONCLUSION}

La edad y manejo productivo de cacaotal es un factor limitante en la diversidad de especies. Así mismo la asociación con otras especies (específicamente frutales y maderables) no solo crea diversidad hablando ecológicamente si no que representa una alternativa viable para mejorar la calidad del aire, suelo y beneficios económicos para el productor.

Con respecto a cantidad de biomasa en t/ha-1 hay que tener en cuenta que las variables diámetro, altura y densidad de madera de las especies arbóreas, determina en gran medida el contenido de carbono. Así mismo el carbono encontrado en el suelo (raíz, Hojarasca y humus) contribuyen al aumento de la cantidad de carbono para los Sistemas Agroforestales.

El nivel de rentabilidad están determinados por factores como son: el rendimiento productivo $(\mathrm{kg} / \mathrm{ha})$ y los costos de producción (mano de obra e insumos). Mientras mayor sea el costo de producción menor será la rentabilidad de esta y viceversa.

\section{AGRADECIMIENTOS}

A CONACYT por la beca otorgada de posgrado, al Laboratorio de Sistemas de Información Geográfica de la DACBiol, a los productores de Cacao del poblado C-34 por la facilidad de sus parcelas los cuales hicieron posible la realización del este proyecto y a la Línea Prioritaria de Investigación "8" del colegio de Postgraduados "Impacto y Mitigación del Cambio Climático" por el apoyo brindado para el desarrollo de esta investigación.

\section{LITERATURA CITADA}

Alegre J. A.; Ricse A. L.; Barbaran J. P. C. 2001. Reservas de Carbono y emisión de gases con diferentes sistemas de uso de la tierra en dos sitios de la Amazonía peruana. Symposium Internacional de Agroforesteria. Manaus, Brazil EMBRAPA 21-24 de Noviembre 2000.

Beer J.; Harvey C.; Ibrahim M.; Harmand J. M.; Somarriba E., Jiménez, F. 2003. Servicios ambientales de los sistemas agroforestales. Agroforestería en las Américas 10(37-38): 80-87.

Brown S., 1997. Estimating biomass and biomass changes of tropical forests: Departtment of NAtural resources enad Environments Sciencies. University of Illinois, Urbana Illinois. A Forestry Resources Assesmente publication. FAO Forestry paper 134

Cairns M. A.; Brown S.; Eileen H. Herlmer; Baumgardner E. H. 1997. Root biomass allocation in the world's upland forest. Oecologia 111: 1-11 https://doi.org/10.1007/s00442005020 PMid:28307494

Carrascosa M. 1995. Análisis Económico y Financiero de la Adopción de Sistemas Agroforestales en San Martin Jilotepeque para la Especie Forestal Alnus Acuminata. Universidad Francisco Marroquín Facultad de Ciencias Económicas. Guatemala. 1-2.

Cartagena M. 2010. "Estudio socio-económico de la producción de cacao orgánico en la zona de pasaje, del canton ventanas, provincia de los ríos". Guayaquil- Ecuador. 29 p.

Callo Concha D. L.; Krishnamurthy J. Alegre 2001. Cuantificación de carbono secuestrado por algunos SAF's testigos, en tres pisos ecológicos de la Amazonía del Perú. Simposio Internacional: Medición y Monitoreo e la Captura de Carbono en Ecosistemas Forestales, 18 al 20 de Octubre del 2001. Valdivia Chile. 19-32. 
Chave J.; Andalo C.; Brown S.; Cirns M.A.; Chambers J. Q.; Eamus D.; Folster H.; Fromard F.; Higuchi N.; Kira T.; Lescure J. P.; Nelson B.W., Ogawa H.; Puig H.; Riera B.; Yamakura T. 2005 Tree allometry and improved estimation of carbon stocks and balance in tropical forests Oecologia 145 87-99

CONABIO 2006. Capital Natural y Bienestar Social. Comisión Nacional para el Conocimiento y uso de la biodiversidad, México, D.F.

Córdova-Ávalos, Sánchez-Hernández M.; Estrella-Chulím N.; Sandoval-Castro E.; Martínez- Saldaña T.; OrtizGarcía C. F. 2001. Factores que Afectan la Producción de Cacao Theobroma cacao L. En el Ejido Francisco I. Madero. Plan Chontalpa, Tabasco, México. Universidad y Ciencia 34 017: 93-100.

Comisión Nacional de Áreas Naturales Protegidas (CONANP). (2001). Programa de Manejo Reserva de la Biósfera Mariposa Monarca. Obtenido de http://www.conanp.gob.mx/que_hacemos/pdf/programas_manejo/ monarca.pdf

Dixon R. K.; Schroeder P. E.; Winjum J. K. 1991. Assessment of promising forest management practices and technologies for enhancing the conservation and sequestration of atmospheric carbon and their costs at the site level. Corvallis, Or EUA. 100 p.

FAO, 2003. Agricultural Transformation and Gender Considerations in Caribbean Economies (documento de trabajo), noviembre de 2003.

García E. 1988. Modificaciones al sistema de clasificación climática de Koeppen'. UNAM. Facultad de Ciencias. México, D.F. 216 p.

García-Yanes M. 1994. El Cacao: Origen, Cultivo e industrialización en Tabasco. Centro de Investigación de Ciencias Agropecuarias. 14-15.

González V. 2005. Cacao en México: Competitividad y Medio Ambiente con alianzas INIFAP y IPRC para USAID. $45 \mathrm{p}$.

Hairiah, K., D. Suprayogo, Widianot, and C. Prayogo. 2005. Trees that produce mulch layers which reduce run-off and soil loss in coffee multistrata systems. Available online at: http://www.icraf.cgiar.org/sea/Publications/files/ bookchapter/ BC0150-05.pdf.

INEGI Instituto Nacional de Estadística, Geografia e Informática 2001. Síntesis Geográfica, Nomenclátor y Anexo Cartográfico del Estado de Tabasco. Instituto nacional de Estadística, Geografía e Informática. México. 69 pp.

IPCC, 2003: Good Practice Guidance for Land Use, Land-Use Change and Forestry. Institute for Global Environmental Strategies (IGES), Hayama, Japan, ISBN 4-88788-003-0.

Lapeyre T.; Alegre J.; Arévalo L. 2004. Determinación de las Reservas de Carbono de la Biomasa Aérea en diferentes sistemas de uso de tierra en San Martín, Perú. Ecología Aplicada 3 1,2: 35-44. https://doi.org/10.21704/ rea.v3i1-2.269

López Báez O.; Huerta, G. 1988. Manual del cacao. Instituto Nacional de Investigaciones Forestales, Agrícolas y Pecuarias. INIFAP. México. 96 pp.

Nair P. K.; Kumar B. M.; Nair V. D. 2009. Agroforestry as a strategy for carbon sequestration. Journal of Plant Nutrition and Soil Science, 172: 10-23. https://doi.org/10.1002/jpln.200800030

Orrala R. 2007.. Almacenamiento de Carbono en dos Sistemas Silvopastoriles Instalados en Quevedo Provincia de los Ríos. Costa Rica. www.dspace.espol.edu.ec/bitstream/123456789/859/1/1592.pdf; 15 de Junio del 2011

Ortiz E. 2002. Sistema de Cobro y Pago de Servicos Ambientales en Costa Rica. Area de Servicios Ambientales, Escuela de Ingeniería Forestal, ITCR. Serie de Apoyo Académico No. 34.

Ortiz Pérez M. A.; Siebe C.; Cram S. 2005. Diferenciación Geográfica de Tabasco, Cap. 14:305-322. En Bueno, J., F. Álvarez y S. Santiago Eds. Biodiversidad del Estado de

Reitsma R. ; Parrish J.D.; McLaney W. 2001. The role of cacao plantations in maintaining forest avian diversity in Southeastern Costa Rica. Agrofor. Syst. 53: 185-193. https://doi.org/10.1023/A:1013328621106

Rendón-Carmona N.; Soto-Pinto L. 2007. Manual de metodología rápida para la estimación y monitoreo de captura de carbono. ECOSUR, Chiapas. 47 pp 
Sánchez-Gutiérrez F.; Pérez F. J.; Obrador O. J. J.; Sol S. A. y Ruiz R. O. 2012. Recursos Maderables en el sistema agroforestal cacao en Cadenas Tabasco. En: Recursos Maderables en el sistema agroforestal cacao en Cadenas Tabasco. Tesis de Maestría en Ciencias. Colegio de Postgraduados 77 pág. Mexico D.f.

SERMARNAT 2006. Secretaria de Medio Ambiente y Recursos Naturales La Gestión Ambiental en México. SEMARNAT, México, D.F

Silva, A. da. 2001. Consideraciones sociales y silviculturales para el manejo forestal diversificado en una comunidad rivereña de la Floresta Nacional do Tapajós, Amazonia Brasileña. Tesis MAg. SC. Turrialba. Costa Rica. CATIE $125 \mathrm{p}$.

Somarriba, E. y Trujillo, L. 2005. El Proyecto Modernización de la cacaocultura del Alto Beni, Bolivia. Agroforestería en las Américas (43/44).

Yuliasmara F.; Wibawa A.; y Prawoto A. 2009. Carbon Stock in Different Ages and Plantation System of Cocoa. Allometric Approach. Pelita Perkebunan: Coffee and Cocoa Research Journal 25(2), 86-100 https:// doi.org/10.22302/iccri.jur.pelitaperkebunan.v25i2.132

Zak J. C.; Willig M. R. 2004. Fungal Biodiversity patterns. in: Mueller G. M., Bills G. F. y Foster, M. S. 2004. Biodiversity of fungi: Inventory and monitoring methods. Elsevier, Academic Press, Amsterdam. 2004. p. 59-75. https://doi.org/10.1016/B978-012509551-8/50008-8

Zanne A. E.; López-González G.; Coomes D. A.; Ilic J. Jansen S.; Lewis S. L., Miller R. B.; Swenson N. G.; Wiemann M. C.; Chave J. 2009. Global wood density database. consultado en línea: http://datadryad.org/handle/10255/ dryad.235 